\title{
OPEN Multicentre, retrospective study of the efficacy and safety of nivolumab for recurrent and metastatic salivary gland carcinoma
}

\author{
Kazutomo Niwa ${ }^{1,2,10}$, Daisuke Kawakita ${ }^{3,10}$, Toshitaka Nagao ${ }^{4}$, Hideaki Takahashi' ${ }^{2}$, \\ Takashi Saotome ${ }^{5}$, Masashi Okazaki ${ }^{6}$, Keisuke Yamazaki ${ }^{7}$, Isaku Okamoto ${ }^{8}$, Hideaki Hirai ${ }^{4}$, \\ Natsuki Saigusa ${ }^{4}$, Chihiro Fushimi ${ }^{1}$, Tatsuo Masubuchi ${ }^{1}$, Kouki Miura ${ }^{1}$, Shin-ichi Okazaki ${ }^{6}$, \\ Hirooki Matsui ${ }^{6}$, Takuro Okada ${ }^{8}$, Sho Iwaki ${ }^{3}$, Takashi Matsuki ${ }^{9}$, Kenji Hanyu ${ }^{1}$, \\ Kiyoaki Tsukahara ${ }^{8}$, Nobuhiko Oridate ${ }^{2}$ \& Yuichiro Tada ${ }^{1,10 \bowtie}$
}

\begin{abstract}
Although immune-checkpoint inhibitors (ICls) are effective against various cancers, little is known regarding their role in salivary gland carcinoma (SGC) treatment. Therefore, we evaluated the efficacy and safety of nivolumab monotherapy in patients with recurrent and/or metastatic SGC. In this multicentre retrospective study, nivolumab $(240 \mathrm{mg})$ was administered every 2 weeks. The overall response rate (ORR), progression-free survival (PFS), overall survival (OS), and safety were examined; the correlation between treatment outcomes and clinicopathological factors was analysed. Twentyfour patients were enrolled; the most common histopathology was salivary duct carcinoma. Eleven tumours were PD-L1-positive; no tumour was microsatellite instability-high. The ORR was $4.2 \%$, and the median PFS and OS were 1.6 and 10.7 months, respectively. One patient continued nivolumab for 28 months without disease progression. One patient showed grade 4 increase in creatine phosphokinase levels and grade 3 myositis. Biomarker analysis revealed significantly increased OS in patients with performance status of 0 ; modified Glasgow prognostic score of 0 ; low neutrophil-tolymphocyte ratio, lactate dehydrogenase, and C-reactive protein; and high lymphocyte-to-monocyte ratio and in patients who received systemic therapy following nivolumab. Although nivolumab's efficacy against SGC was limited, some patients achieved long-term disease control. Further studies are warranted on ICl use for SGC.
\end{abstract}

Salivary gland carcinoma (SGC) is a rare type of cancer accounting for only $0.14 \%$ of all malignant neoplasms; it is estimated that 1.4 in 100,000 individuals are diagnosed with SGC per year ${ }^{1}$. According to the histological classification of salivary gland tumours by the World Health Organization, there are 20 histopathological types of SGC; their prognosis and biological characteristics considerably vary with the histological type ${ }^{2}$. Resection is the standard treatment for SGC regardless of the histopathological type and postoperative radiotherapy is

\footnotetext{
${ }^{1}$ Department of Head and Neck Oncology and Surgery, International University of Health and Welfare, Mita Hospital, Tokyo 108-8329, Japan. ${ }^{2}$ Department of Otorhinolaryngology, Head and Neck Surgery, Yokohama City University, School of Medicine, Yokohama, Kanagawa 236-0004, Japan. ${ }^{3}$ Department of Otorhinolaryngology, Head and Neck Surgery, Nagoya City University Graduate School of Medical Sciences, Nagoya 467-8602, Japan. ${ }^{4}$ Department of Anatomic Pathology, Tokyo Medical University, Tokyo 160-0023, Japan. ${ }^{5}$ Division of Medical Oncology, Matsudo City Hospital, Chiba 270-2252, Japan. ${ }^{6}$ Department of Otorhinolaryngology, Head and Neck Surgery, Nihonkai General Hospital, Yamagata 998-8501, Japan. ${ }^{7}$ Department of Otolaryngology Head and Neck Surgery, Niigata University Graduate School of Medical and Dental Sciences, Niigata 951-8520, Japan. ${ }^{8}$ Department of Otorhinolaryngology Head and Neck Surgery, Tokyo Medical University, Tokyo 160-0023, Japan. ${ }^{9}$ Department of Otorhinolaryngology, Head and Neck Surgery, Kitasato University School of Medicine, Sagamihara 252-0375, Japan. ${ }^{10}$ These authors contributed equally: Kazutomo Niwa, Daisuke Kawakita and Yuichiro Tada. ${ }^{\circledR}$ email: ytada@iuhw.ac.jp
} 
recommended for patients at a high risk of recurrence ${ }^{3}$. Numerous clinical trials of cytotoxic chemotherapies have been conducted in patients with recurrent and/or metastatic (R/M) SGC ${ }^{3,4}$ and several potential targets for systemic therapy have been reported ${ }^{5-17}$; however, there were no randomised controlled trials ${ }^{3,4}$. Moreover, unlike lung metastasis of adenoid cystic carcinoma (AdCC), majority of which show indolent growth ${ }^{3,18,19}$, the progression of salivary duct carcinoma (SDC) and adenocarcinoma, not otherwise specified (NOS) is aggressive ${ }^{3,20,21}$. Hence, there is a need for systemic therapeutic strategies based on the histological characteristics for SGC.

Immune-checkpoint inhibitors (ICIs) have demonstrated durable antitumor effects against multiple cancer types, including head and neck squamous cell carcinoma ${ }^{22,23}$. Two prospective studies on pembrolizumab monotherapy $y^{24}$ and pembrolizumab combined with vorinostat ${ }^{25}$ for SGC have been published. Furthermore, two studies on nivolumab in patients with non-HNSCC, including $\operatorname{six}^{26}$ and two ${ }^{27}$ patients with SGC, respectively, have been published. However, these studies were not conducted exclusively in patients with SGC; to the best of our knowledge, there are no studies on the efficacy of nivolumab for SGC.

As a significant number of patients fail to benefit from ICIs; studies have been conducted to identify biomarkers to predict the response of patients to ICIs. Programmed death-ligand 1 (PD-L1) expression $22,23,28,29$ and microsatellite instability (MSI) ${ }^{28,30}$ are used as companion diagnostic markers; tumour mutation burden

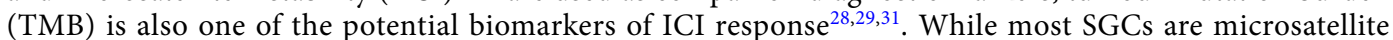
stable $^{32-34}$, there are no consistent data on PD-L1 expression in SGCs due to the use of different antibodies and evaluation methods among studies ${ }^{35-39}$. Several case series studies of PD-L1 expression in SGCs including multiple histopathological types have showed a significantly higher positivity of PD-L1 in SDC than in other histopathological types of SGC ${ }^{35-39}$. Moreover, SDC is reported to harbour a higher mutational burden than other types of SGC $32,34,39-41$. Overall, these findings suggest that ICIs may have a higher efficacy against SDCs than other histopathological types of SGCs.

Recently, inflammatory markers including neutrophil-to-lymphocyte ratio (NLR), platelet-to-lymphocyte ratio (PLR), lymphocyte-to-monocyte ratio (LMR), serum C-reactive protein (CRP), modified Glasgow prognostic score (mGPS), absolute eosinophil count, and serum lactate dehydrogenase (LDH) have been reported to be useful predictors of progression of cancer including SDC ${ }^{42,43}$. Although it is largely unknown how systemic inflammation affects the survival of patients with cancer, neutrophils are known to promote tumour growth and distant metastasis by releasing cytokines that promote neovascularisation. As the number of lymphocytes reflects antitumor immunity, increased NLR due to neutrophilia and lymphocytopenia is associated with worse prognosis in patients treated with ICIs ${ }^{44-46}$. The LMR, PLR, CRP level, mGPS, absolute eosinophil count, and LDH have also been reported to correlate with the therapeutic effects of ICIs in patients with melanoma, non-small-cell lung cancer, and head and neck squamous cell carcinoma ${ }^{46-51}$. Therefore, in this retrospective multicentre study, we aimed to evaluate the efficacy of nivolumab monotherapy in patients with SGC including SDC. Additionally, we conducted a database analysis to determine the correlation between clinical profiles including PD-L1 positivity, MSI, and inflammatory biomarkers and the survival of patients with SGC treated with nivolumab.

\section{Results}

Patient characteristics and treatment. Twenty-four patients, identified in the cancer registry of the participating institutions during the study period, were enrolled in this study (Table 1). Written informed consent was obtained from all participants. The median follow-up period for all patients was 6.5 (range 0.6-28.2) months. All patients had received systemic therapy before nivolumab. The most common histopathological type of cancer was SDC $(n=20,83 \%)$. Eleven tumours (46\%) presented PD-L1 expression at a rate of $\geq 1 \%$. Among them, three (13\%) presented $\geq 50 \%$ PD-L1 positivity. Among 23 evaluable patients, none was classified as MSIH.

The median number of cycles of nivolumab administered was 8 (range 1-57). As of the cut-off date, 30 January 2020, two patients (8\%) continued to receive nivolumab for 28 and 6 months, whereas 22 patients (92\%) discontinued treatment due to PD $(n=19,79 \%)$ and AEs $(n=3,13 \%)$. Six patients $(25 \%)$ received one or more of the following systemic therapy regimens after nivolumab treatment: cetuximab plus paclitaxel $(n=5,21 \%)$, carboplatin plus docetaxel, trastuzumab plus docetaxel, abiraterone, and S-1 $(n=1,4 \%$, respectively).

Response and survival outcomes. The therapeutic efficacy of nivolumab are shown in Table 2. None of the patients achieved CR; 1 (4\%), 2 (8\%), and 21 (88\%) patients showed PR, SD, and PD, respectively. The ORR was $4.2 \%$ (95\% CI $0.1-21.1 \%)$. Two patients with SD maintained the status for more than 24 weeks. Thus, both CBR and DCR were $12.5 \%$ (95\% CI 2.7-32.4\%). The Kaplan-Meier survival curves of PFS and OS of all patients are shown in Fig. 1; the median PFS was 1.6 (95\% CI 1.2-4.4) months and the median OS was 10.7 (95\% CI 5.1-19.8) months. The therapeutic effects observed in 20 patients with SDC were as follows: ORR, 5.0\% (95\% CI 2.7-24.9\%); median PFS, 1.5 (95\% CI 1.1-2.7) months; and median OS, 11.3 (95\% CI 3.8-19.8) months. Figure 2 shows the waterfall, spider, and swimmer plots of all patients based on the histopathological diagnosis. Figure 3 shows the representative images of tumour before and during nivolumab monotherapy in two patients.

Safety. All AEs reported are listed in Table 3. Twenty-two patients (92\%) experienced at least one AE during the treatment. Six patients $(25 \%)$ showed grade 3 or 4 AEs; 1 patient showed grade 4 increase in creatine phosphokinase (4\%); 3 patients (13\%) showed grade 3 anaemia, and 1 patient each showed an increase in alkaline phosphatase, amylase, aspartate aminotransferase, alanine transaminase, hyponatraemia, and myositis (4\%). No treatment-related death was observed. Frequent AEs of all grades included anaemia $(n=17,71 \%)$, increased alkaline phosphatase $(n=10,42 \%)$, and hypoalbuminemia $(n=9,38 \%)$. Five patients $(21 \%)$ had an irAE, and only one of these patients $(4 \%)$ had grade 4 increase in creatine phosphokinase and grade 3 myositis. Other 
n (\%)

Age (years)

Median (range)

$56(29-82)$

Sex

\begin{tabular}{|l|l}
\hline Male & $19(79)$ \\
\hline Fen & $5(21)$
\end{tabular}

Female

$5(21)$

Primary site

Parotid gland

$19(79)$

\begin{tabular}{l|l}
\hline Submandibular gland & $3(13)$
\end{tabular}

Minor salivary gland

Accessory parotid gland

$1(4)$

Histopathology

\begin{tabular}{|l|l|}
\hline Salivary duct carcinoma & $20(83)$
\end{tabular}

\begin{tabular}{|l|l|}
\hline Adenocarcinoma, NOS & $2(8)$
\end{tabular}

\begin{tabular}{|l|l|}
\hline Adenoid cystic carcinoma & $1(4)$ \\
\hline
\end{tabular}

\begin{tabular}{|l|l}
\hline Mucoepidermoid carcinoma & $1(4)$
\end{tabular}

Immunohistochemistry

\begin{tabular}{|l|l}
\hline HER2-positive $^{\mathrm{a}}$ & $11(46)$ \\
\hline
\end{tabular}

\begin{tabular}{|l|l|}
\hline AR-positive & 20 (83) \\
\hline
\end{tabular}

\begin{tabular}{|l|l}
\hline PD-L1 $(28-8)<1 \%$ & $13(54)$
\end{tabular}

\begin{tabular}{|l|l|}
\hline PD-L1 (28-8) 1-9\% & $5(21)$ \\
\hline
\end{tabular}

\begin{tabular}{|l|l|}
\hline PD-L1 (28-8) 10-49\% & $3(13)$ \\
\hline
\end{tabular}

\begin{tabular}{|l|l|}
\hline PD-Ll $(28-8) \geq 50 \%$ & $3(13)$
\end{tabular}

\begin{tabular}{|l|l}
\hline MSI-H $(\mathrm{n}=23)$ & $0(0)$
\end{tabular}

Prior treatment

\begin{tabular}{|l|l|}
\hline None & $0(0)$ \\
\hline
\end{tabular}

\begin{tabular}{|l|l|}
\hline Surgery & $18(75)$ \\
\hline
\end{tabular}

\begin{tabular}{|l|l|}
\hline Radiotherapy & $19(79)$ \\
\hline
\end{tabular}

\begin{tabular}{l|l} 
Concomitant radiotherapy (cisplatin) & $9(38)$
\end{tabular}

Concomitant radiotherapy (carboplatin) $2(8)$

\begin{tabular}{|l|l|}
\hline Systemic therapy & $24(100)$
\end{tabular}

\begin{tabular}{|l|l}
\hline Systemic therapy for RM disease & $22(100)$
\end{tabular}

\begin{tabular}{l|l|l}
\hline Leuprorelin + bicalutamide & $10(42)$
\end{tabular}

\begin{tabular}{l|l} 
Carboplatin + paclitaxel & 7 (29)
\end{tabular}

\begin{tabular}{l|l} 
Trastuzumab + docetaxel & $7(29)$
\end{tabular}

\begin{tabular}{l|l} 
Carboplatin + docetaxel & $5(21)$
\end{tabular}

\begin{tabular}{l|l} 
S-1 & $5(21)$
\end{tabular}

\begin{tabular}{l|l} 
Trastuzumab + S-1 & $5(21)$
\end{tabular}

\begin{tabular}{l|l} 
Others $^{c}$ & $13(54)$
\end{tabular}

\begin{tabular}{|l|l}
\hline Systemic therapy after nivolumab & $6(25)$
\end{tabular}

\begin{tabular}{l|l} 
Cetuximab + paclitaxel & $5(21)$
\end{tabular}

\begin{tabular}{l|l} 
Trastuzumab + docetaxel & $1(4)$
\end{tabular}

\begin{tabular}{l|l} 
Carboplatin + docetaxel & $1(4)$
\end{tabular}

\begin{tabular}{l|l} 
Abiraterone & $1(4)$
\end{tabular}

\begin{tabular}{l|l} 
S-1 & $1(4)$
\end{tabular}

\begin{tabular}{|l|l|}
\hline Platinum refractory & $12(50)$ \\
\hline
\end{tabular}

Target lesion

\begin{tabular}{|l|l|}
\hline Locoregional & 4 (17)
\end{tabular}

\begin{tabular}{|l|l|}
\hline Locoregional + distant metastasis & $1(4)$ \\
\hline
\end{tabular}

\begin{tabular}{l|l} 
Distant metastasis only & $19(79)$
\end{tabular}

Site of metastasis

\begin{tabular}{|l|l|}
\hline Lung & $10(42)$ \\
\hline Liver & $5(21)$ \\
\hline Lymph nodes & $4(17)$ \\
\hline Soft tissue (skin, muscle) & $3(13)$ \\
\hline Bone & $1(4)$ \\
\hline Brain, meninges & $1(4)$ \\
\hline Continued &
\end{tabular}




\begin{tabular}{|l|l|}
\hline & n (\%) \\
\hline Pleura & $1(4)$ \\
\hline Pericardium & $1(4)$ \\
\hline
\end{tabular}

Table 1. Baseline patients' characteristics. $A R$ androgen receptor, $C A B$ combined androgen blockade, $H E R 2$ human epidermal growth factor receptor 2, $m G P S$ modified Glasgow prognostic score, $M S I-H$ high-frequency microsatellite instability, $P D-L 1$ programmed death-ligand $1, R M$ recurrent/metastatic, $S D C$ salivary duct carcinoma, TPF docetaxel/cisplatin/5-fluorouracil. ${ }^{a}$ The HER2 status was defined according to the American Society of Clinical Oncology/College of American Pathologists (ASCO/CAP) guidelines for breast cancer ${ }^{54}$. ${ }^{\mathrm{b}}$ A case was considered to be AR-positive when $\geq 20 \%$ of the tumour cell nuclei showed strong staining ${ }^{55}$. ${ }^{\mathrm{c}}$ Abiraterone, 3; bicalutamide, 2; enzalutamide, 2; docetaxel, cisplatin + docetaxel, carboplatin + pemetrexed, cisplatin + 5-fluorouracil, cisplatin + 5-fluorouracil + cetuximab and trastuzumab + docetaxel + pertuzumab, 1 each.

\begin{tabular}{|c|c|c|}
\hline Efficacy & All patients $(n=24)$ & Salivary duct carcinoma $(n=20)$ \\
\hline Complete response, $\mathrm{n}(\%)$ & $0(0)$ & $0(0)$ \\
\hline Partial response, $\mathrm{n}(\%)$ & $1(4.2)$ & $1(5.0)$ \\
\hline Stable disease, $\mathrm{n}(\%)$ & $2(8.3)$ & $0(0)$ \\
\hline Progressive disease, $\mathrm{n}(\%)$ & $21(87.5)$ & $19(95.0)$ \\
\hline Objective response $^{\mathrm{a}}, \mathrm{n}(\%, 95 \% \mathrm{CI})$ & $1(4.2,0.1-21.1)$ & $1(5.0,2.7-24.9)$ \\
\hline Disease control $^{\mathrm{b}}, \mathrm{n}(\%, 95 \% \mathrm{CI})$ & $3(12.5,2.7-32.4)$ & $1(5.0,2.7-24.9)$ \\
\hline Stable disease $\geq 24$ weeks, $\mathrm{n}(\%)$ & $2(8.3)$ & $0(25.0)$ \\
\hline Clinical benefit ${ }^{\mathrm{c}}, \mathrm{n}(\%, 95 \% \mathrm{CI})$ & $3(12.5,2.7-32.4)$ & $1(5.0,2.7-24.9)$ \\
\hline Median progression-free survival, months (95\% CI) & $1.6(1.2-4.4)$ & $1.5(1.1-2.7)$ \\
\hline Median overall survival, months (95\% CI) & $10.7(5.1-19.8)$ & $11.3(3.8-19.8)$ \\
\hline
\end{tabular}

Table 2. Treatment efficacy. CI confidence interval, NR not reached. ${ }^{a}$ Confirmed complete and partial responses. ${ }^{b}$ Complete response, partial response, and stable disease. ${ }^{c}$ Complete response, partial response, and stable disease $\geq 24$ weeks.

irAEs reported were grade 2 pneumonitis $(n=1,4 \%)$, grade 1 hyperthyroidism $(n=2,8 \%)$, and hypothyroidism $(\mathrm{n}=1,4 \%)$. Three patients discontinued treatment due to AEs including grade 3 myositis $(\mathrm{n}=1)$ and grade 2 pneumonitis $(\mathrm{n}=2)$.

Exploratory analysis of biomarkers of $\mathrm{ICI}$ response. Table 4 and Fig. 4 show the results of the exploratory analysis of the biomarkers. As all patients were microsatellite stable, no analysis was performed according to the MSI status. There was no association between PD-L1 positivity and prognosis. In the univariate analysis, higher NLR, higher serum LDH and CRP, and lower LMR were significantly associated with shorter PFS. The significant predictors of a shorter OS were the ECOG PS $\geq 1, \mathrm{mGPS} \geq 1$, higher NLR, higher serum $\mathrm{LDH}$, and higher serum CRP. Systemic therapy following nivolumab and higher LMR were significantly associated with a longer OS. The Kaplan-Meier curves of the OS and PFS, waterfall, spider, and swimmer plots according to the biomarkers are presented in Fig. 3, Supplementary Figs. S1 and S2, respectively. The 1-year OS of patients with the ECOG PS 0, with systemic therapy after nivolumab, mGPS 0, lower NLR, higher LMR, lower LDH level, and lower CRP level was 59.3\%, 83.3\%, 65.0\%, 85.7\%, 52.5\%, 55.5\%, and 68.6\%, respectively (Fig. 3).

\section{Discussion}

In the present retrospective study of nivolumab monotherapy in 24 patients with R/M SGC, the ORR was $4.2 \%$, with the median PFS and OS of 1.6 and 10.7 months, respectively. The ORR of the 20 patients with SDC was $5.0 \%$ and the median PFS and OS were 1.5 and 11.3 months, respectively. Nivolumab was well tolerated by patients with SGC, and AEs associated with nivolumab was comparable with those associated with pembrolizumab ${ }^{24}$. In this study, the therapeutic effects were limited; however, some patients achieved considerably long-term disease control.

Prospective studies on pembrolizumab ${ }^{24}$ and pembrolizumab combined with vorinostat ${ }^{25}$ reported that the ORR of patients with multiple histopathological types of SGC was $12 \%$ and $16 \%$, respectively, with the median PFS and OS of 4-6.9 and 13-14 months, respectively. The ORR and the median OS of patients with SGC to nivolumab in this study were comparable with those observed in patients with advanced SGC treated with pembrolizumab ${ }^{24,25}$. However, the median PFS of this study was shorter than that reported by the previous study on pembrolizumab. This could be because only patients with obvious progression within 6 months were included in this study, whereas the previous studies with pembrolizumab did not adopt this criterion.

In the present study, the results of the biomarker analysis revealed that most factors related to PS and inflammatory biomarkers such as the NLR, LMR, LDH, and CRP levels were associated with the prognosis of tumour in 
A

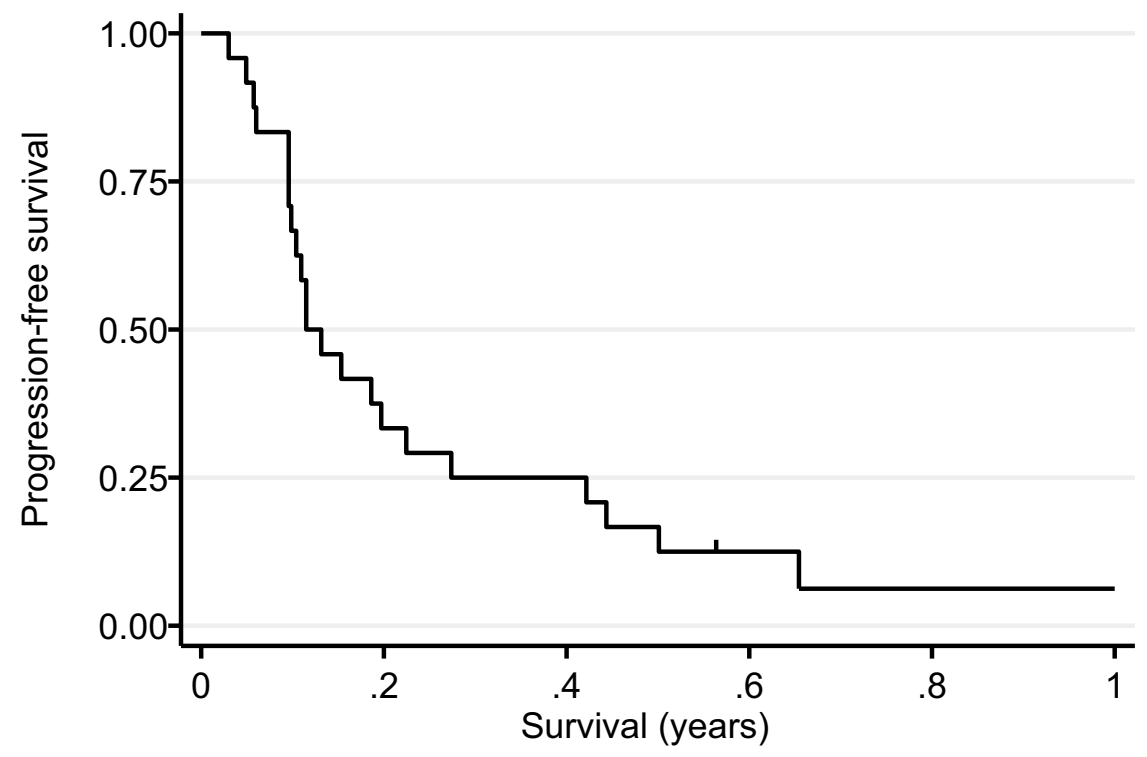

Number at risk 24

8

6

2

1

B

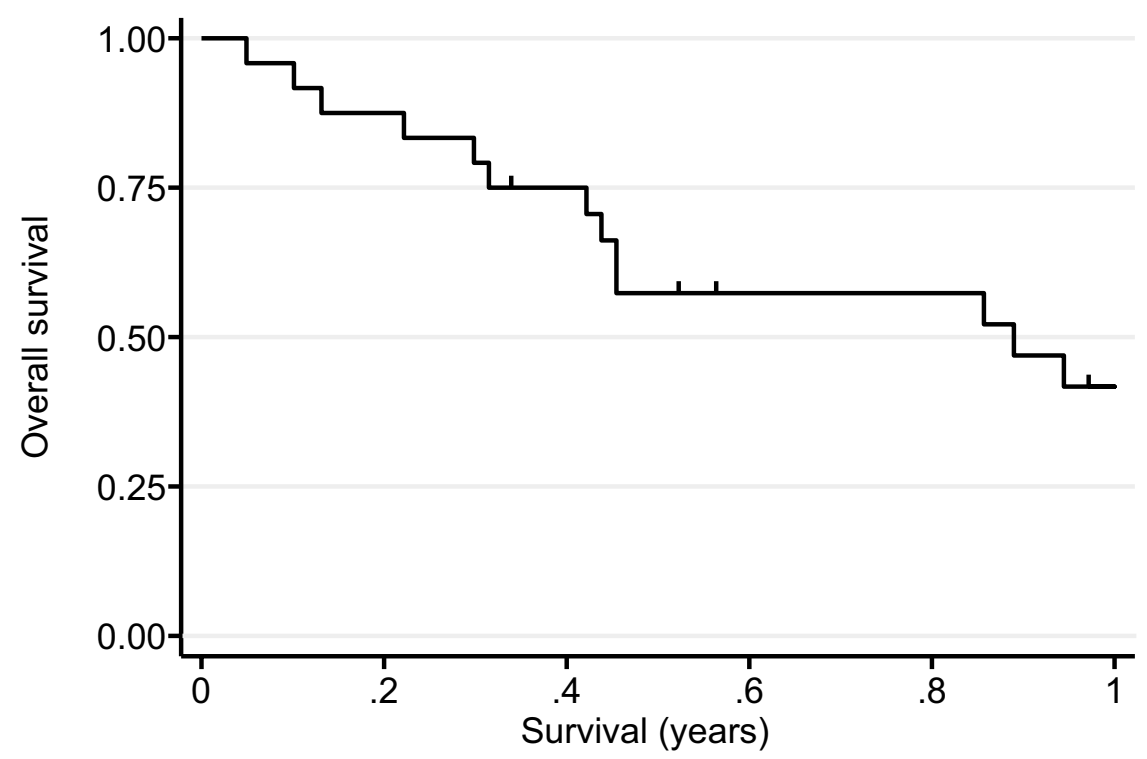

Number at risk 24

21

17

11

11

7

Figure 1. Kaplan-Meier curves of progression-free and overall survival. Kaplan-Meier curves of (A) progression-free survival and (B) overall survival. The vertical lines indicate censored events.

patients treated with nivolumab. To the best of our knowledge, this is the first study to demonstrate an association between inflammatory biomarkers and prognosis in patients with SGC treated with nivolumab. In particular, NLR showed an apparent negative dose-response relationship with the OS; the 1-year OS of patients with lower NLR was $85.7 \%$. The NLR, LMR, PLR, CRP level, mGPS, and LDH level have also been reported to correlate with the therapeutic effects of ICIs in various cancers ${ }^{46-51}$. As a result, NLR, LMR, PLR, LDH, CRP, and mGPS are suggested to reflect the general conditions (immunological competence) of the host, and they can be used as biomarkers of ICI treatment response.

In malignant melanoma, non-small cell lung cancer, and head and neck squamous cell carcinoma, PD-L1 immunohistochemistry $y^{22,23,28,29}$ including the presence of PD-L1-expressing immune cells in the tumour microenvironment ${ }^{52,53}$, mismatch repair (MMR)/MSI ${ }^{29,31}$, and $\mathrm{TMB}^{29-31}$ have been reported as biomarkers of ICI response. Based on the findings of previous studies, which showed significantly higher PD-L1 expression and 


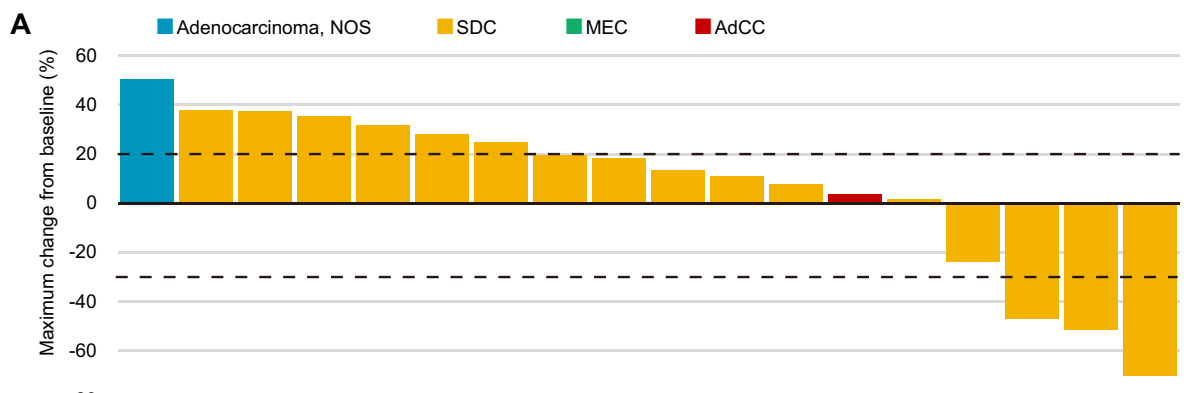

\section{B}

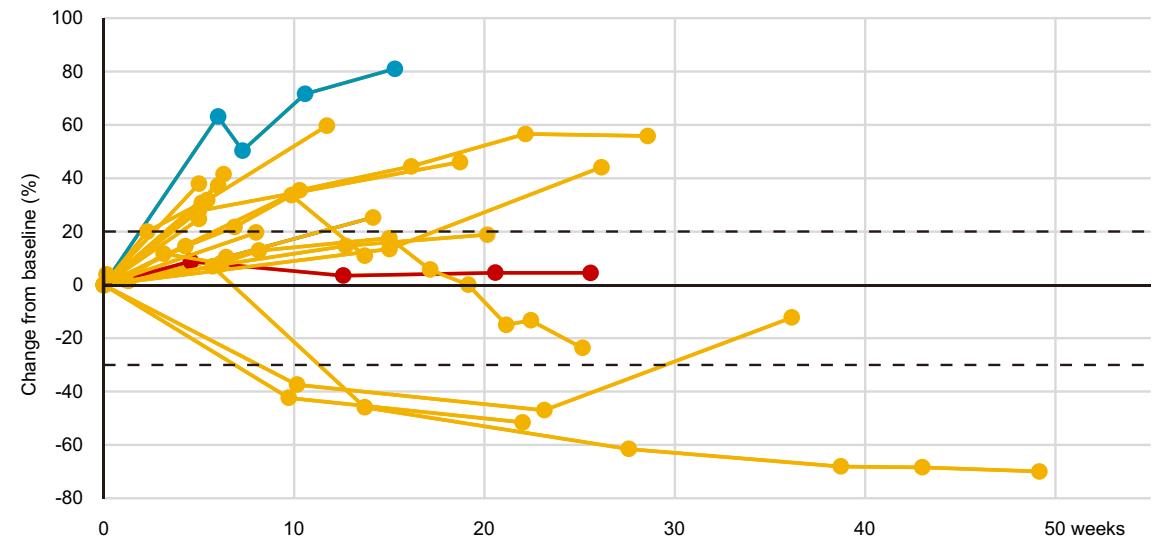

C



Figure 2. Characteristics of responses in patients with salivary gland carcinoma treated with nivolumab according to Response Evaluation Criteria in Solid Tumours (version 1.1) based on histopathological diagnosis. (A) The highest reduction from the baseline in target lesions. Tumour shrinkage relative to the baseline was observed in four patients (16.7\%). The upper dotted lines represent the threshold for progressive disease (a $20 \%$ increase in the sum of the longest diameter of the target lesions) and the lower dotted lines represent the threshold for a partial response (a 30\% decrease in the sum of the longest diameter of the target lesions). (B) Change from the baseline (\%) in the sum of the target lesions over time to progressive disease. The upper dotted lines represent the threshold for progressive disease (a 20\% increase in the sum of the longest diameter of the target lesions) and the lower dotted lines show the threshold for a partial response (a 30\% decrease in the sum of the longest diameter of the target lesions). (C) Time to response and the duration of survival. Each bar represents an individual patient, with the length of the bar corresponding to the time of overall survival based on the disease status. SDC salivary duct carcinoma, $M E C$ mucoepidermoid carcinoma, AdCC adenoid cystic carcinoma. 

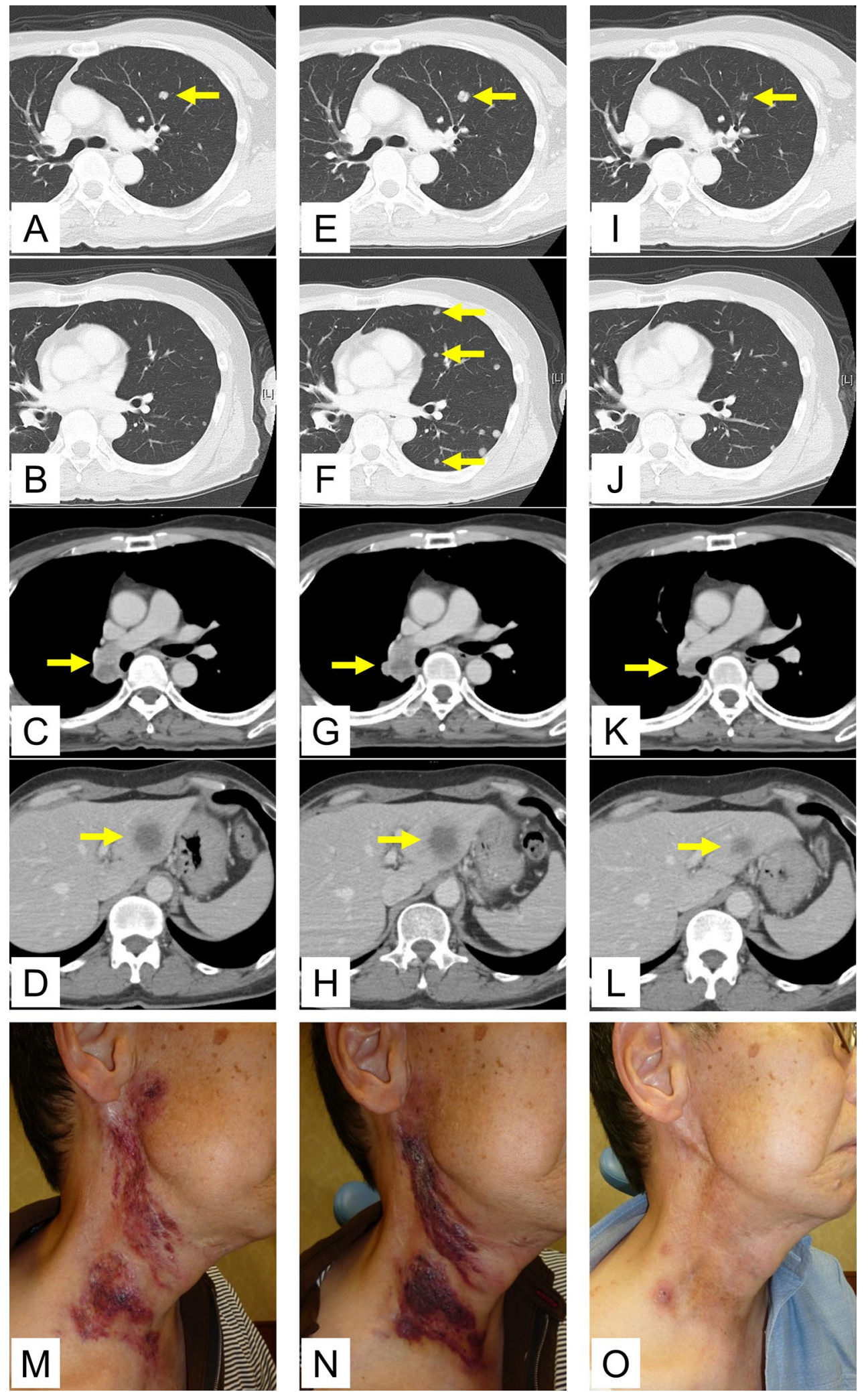

Figure 3. Representative images of the tumor before and during nivolumab monotherapy in two patients with recurrent and/ or metastatic salivary gland carcinoma. (A-D) Pre-treatment of a patient with lung, liver, and hilar lymph node metastasis, (E-H) 40 days after the initiation of nivolumab treatment, (I-L) 96 days after the initiation of nivolumab treatment. Tumor shrinkage was observed in this patient following PD diagnosis due to a new lesion. The yellow arrows indicate lung metastases (A, E, I), new lung lesion (F), hilar lymph node metastasis $(\mathbf{C}, \mathbf{G}, \mathbf{K})$, and liver metastases (D, H, L). (M-O) Pre-treatment of a patient with cervical skin metastasis, (P-R) 57 days after the initiation of nivolumab treatment, (S-U) 204 days after the initiation of nivolumab treatment. Tumor shrinkage was observed in this patient after an increase in skin tumor thickness, which was in the SD range. 


\begin{tabular}{|c|c|c|c|c|c|}
\hline Event & Any grade & Grade 1 & Grade 2 & Grade 3 & Grade 4 \\
\hline Any & $22(92)$ & $19(79)$ & $8(33)$ & $6(25)$ & $1(4)$ \\
\hline Anaemia & $17(71)$ & $11(46)$ & $3(13)$ & $3(13)$ & 0 \\
\hline ALP increased & $10(42)$ & $8(33)$ & $1(4)$ & $1(4)$ & 0 \\
\hline Hypoalbuminemia & $9(38)$ & $7(29)$ & $2(8)$ & 0 & 0 \\
\hline Hyperkalaemia & $6(25)$ & $3(17)$ & $3(13)$ & 0 & 0 \\
\hline Heart failure & $6(25)$ & $6(25)$ & 0 & 0 & 0 \\
\hline Serum amylase increased & $5(21)$ & $3(13)$ & $1(4)$ & $1(4)$ & 0 \\
\hline AST increased & $5(21)$ & $4(17)$ & 0 & $1(4)$ & 0 \\
\hline Hyponatraemia & $4(17)$ & $3(13)$ & 0 & $1(4)$ & 0 \\
\hline $\mathrm{CPK}$ increased & $3(13)$ & 0 & $2(8)$ & 0 & $1(4)$ \\
\hline ALT increased & $3(13)$ & $2(8)$ & 0 & $1(4)$ & 0 \\
\hline Cre increased & $3(13)$ & $1(4)$ & $2(8)$ & 0 & 0 \\
\hline Chronic kidney disease & $2(8)$ & 0 & $2(8)$ & 0 & 0 \\
\hline$\gamma$-GTP increased & $2(8)$ & $1(4)$ & $1(4)$ & 0 & 0 \\
\hline Pneumonitis & $2(8)$ & 0 & $2(8)$ & 0 & 0 \\
\hline Hyperthyroidism & $2(8)$ & $2(8)$ & 0 & 0 & 0 \\
\hline Hypertriglyceridaemia & $2(8)$ & $2(8)$ & 0 & 0 & 0 \\
\hline Hypokalaemia & $2(8)$ & $2(8)$ & 0 & 0 & 0 \\
\hline APTT prolonged & $2(8)$ & $2(8)$ & 0 & 0 & 0 \\
\hline Blood LDH increased & $2(8)$ & $2(8)$ & 0 & 0 & 0 \\
\hline Hyperglycaemia & $2(8)$ & $2(8)$ & 0 & 0 & 0 \\
\hline Myositis & $1(4)$ & 0 & 0 & $1(4)$ & 0 \\
\hline Lymphocyte count decreased & $1(4)$ & 0 & $1(4)$ & 0 & 0 \\
\hline INR increased & $1(4)$ & 0 & $1(4)$ & 0 & 0 \\
\hline White blood cell decreased & $1(4)$ & $1(4)$ & 0 & 0 & 0 \\
\hline Platelet count decreased & $1(4)$ & $1(4)$ & 0 & 0 & 0 \\
\hline Hypothyroidism & $1(4)$ & $1(4)$ & 0 & 0 & 0 \\
\hline Cholesterol high & $1(4)$ & $1(4)$ & 0 & 0 & 0 \\
\hline Hyperuricaemia & $1(4)$ & $1(4)$ & 0 & 0 & 0 \\
\hline Hypophosphataemia & $1(4)$ & $1(4)$ & 0 & 0 & 0 \\
\hline Arthralgia & $1(4)$ & $1(4)$ & 0 & 0 & 0 \\
\hline
\end{tabular}

Table 3. Reported adverse events, $\mathrm{n}(\%)$. ALP alkaline phosphatase, $A L T$ alanine aminotransferase, $A P T T$ activated partial thromboplastin time, AST aspartate aminotransferase, CPK creatinine phosphatase, $\gamma$-GTP $\gamma$-glutamyl transpeptidase, $L D H$ lactate dehydrogenase, INR international normalised ratio.

TMB in SDC than those in other tumours ${ }^{32-41}$, higher therapeutic effects of ICIs are being expected. However, the efficacy of ICI monotherapy for SDC was limited in our cohort. This might be since no tumour was MSI-H and the PD-L1-positivity rate in the tumour cells was low in our SDC cohort.

While previous studies on anti-HER2 antibody including trastuzumab ${ }^{5-8}$ and androgen deprivation therapy (e.g., bicalutamide and leuprorelin $)^{9-12}$ for HER2- or AR-positive patients with SDC showed the ORR was $20-89 \%$, the response rate of patients to nivolumab in this study was unsatisfactory. Thus, nivolumab monotherapy is not recommended for patients with HER2- or AR-positive advanced SDCs before anti-HER2- or AR-targeted therapy. In contrast, ICIs might be tried in patients with SGC without targetable molecules instead of conventional cytotoxic anticancer agents. Recent studies reported that cytotoxic anticancer agents seemed to achieve a higher ORR with higher toxicity than the ICIs ${ }^{3,4,24,25}$. However, it is difficult to directly compare those therapies as patient background (e.g., histological type) might differ. As our data suggest low nivolumab ORR and shorter survival in patients with increased systemic inflammatory markers (e.g., NLR), the use of cytotoxic anticancer agents may be prioritised in symptomatic patients (e.g., patients with pain and/or aggressive tumour growth) and patients with increased systemic inflammatory markers. Currently, a clinical trial on the combination of pembrolizumab and docetaxel in patients with thyroid cancer or SGC without standardof-care treatment is under progress (ClinicalTrials.gov Identifier: NCT03360890). Other ongoing clinical trials targeting patients with SGC include the combination of pembrolizumab and lenvatinib (ClinicalTrials.gov Identifier: NCT04209660), two ICIs (nivolumab and ipilimumab; ClinicalTrials.gov Identifier: NCT02834013, NCT03146650 and NCT03172624), and ICIs and AR-targeted therapy (pembrolizumab and goserelin acetate; ClinicalTrials.gov Identifier: NCT03942653).

This study had some limitations. First, owing to the retrospective nature and small sample size of the study, the superiority of nivolumab over other drugs was not examined. Second, the biomarkers identified in this study including NLR might be merely prognostic factors, which are associated with survival and might not predict 


\begin{tabular}{|c|c|c|c|c|c|c|c|}
\hline & \multirow[b]{2}{*}{$\mathbf{N}$} & \multicolumn{3}{|c|}{ Progression-free survival } & \multicolumn{3}{|c|}{ Overall survival } \\
\hline & & HR & $95 \% \mathrm{CI}$ & $P$-value & HR & $95 \% \mathrm{CI}$ & $P$-value \\
\hline \multicolumn{8}{|l|}{ Age } \\
\hline$<65$ years & 14 & 1.00 & - & - & 1.00 & - & - \\
\hline$\geq 65$ years & 10 & 1.81 & $0.76-4.30$ & 0.181 & 1.05 & $0.37-2.96$ & 0.931 \\
\hline \multicolumn{8}{|l|}{ Sex } \\
\hline Male & 19 & 1.00 & - & - & 1.00 & - & \\
\hline Female & 5 & 0.53 & $0.15-1.85$ & 0.322 & 1.27 & $0.40-4.00$ & 0.686 \\
\hline \multicolumn{8}{|l|}{ ECOG PS } \\
\hline 0 & 13 & 1.00 & - & - & 1.00 & - & - \\
\hline$\geq 1$ & 11 & 1.99 & $0.85-4.68$ & 0.115 & 2.87 & $1.08-7.61$ & 0.034 \\
\hline \multicolumn{8}{|l|}{ Primary site } \\
\hline Parotid gland & 19 & 1.00 & - & - & 1.00 & - & - \\
\hline Others & 5 & 0.62 & $0.21-1.86$ & 0.393 & 1.04 & $0.33-3.25$ & 0.945 \\
\hline \multicolumn{8}{|l|}{ Prior systemic therapy } \\
\hline- & 2 & 1.00 & - & - & 1.00 & - & - \\
\hline+ & 22 & 2.95 & $0.37-23.28$ & 0.305 & 2.72 & $0.33-22.11$ & 0.350 \\
\hline \multicolumn{8}{|l|}{ irAE } \\
\hline- & 19 & 1.00 & - & - & 1.00 & - & - \\
\hline+ & 5 & 1.99 & $0.70-5.64$ & 0.196 & 2.27 & $0.69-7.47$ & 0.178 \\
\hline \multicolumn{8}{|c|}{ Systemic therapy after nivolumab } \\
\hline- & 15 & 1.00 & - & - & 1.00 & - & - \\
\hline+ & 7 & 0.82 & $0.32-2.08$ & 0.673 & 0.06 & $0.01-0.48$ & 0.008 \\
\hline \multicolumn{8}{|l|}{ Histopathology } \\
\hline Salivary duct carcinoma & 20 & 1.00 & - & - & 1.00 & - & - \\
\hline Others & 4 & 0.36 & $0.08-1.58$ & 0.176 & 0.81 & $0.23-2.88$ & 0.746 \\
\hline \multicolumn{8}{|l|}{ PD-L1 } \\
\hline $0 \%$ & 13 & 1.00 & - & - & 1.00 & - & - \\
\hline$\geq 1 \%$ & 11 & 0.85 & $0.36-2.03$ & 0.716 & 0.73 & $0.25-2.10$ & 0.558 \\
\hline \multicolumn{8}{|l|}{ mGPS } \\
\hline 0 & 14 & 1.00 & - & - & 1.00 & - & - \\
\hline$\geq 1$ & 9 & 2.69 & $0.99-7.30$ & 0.052 & 30.06 & $3.66-246.95$ & 0.002 \\
\hline \multicolumn{8}{|c|}{ Neutrocyte-to-lymphocyte ratio } \\
\hline$<2.6$ & 9 & 1.00 & - & - & 1.00 & - & - \\
\hline $2.6-5.0$ & 8 & 2.04 & $0.72-5.79$ & 0.179 & 8.90 & $1.81-43.80$ & 0.007 \\
\hline \multirow[t]{2}{*}{$5.9-19.8$} & 6 & 4.02 & $1.24-13.07$ & 0.021 & 15.48 & $2.82-85.04$ & 0.002 \\
\hline & & $P_{\text {trend }}=0.023$ & & & $\mathrm{P}_{\text {trend }}=0.001$ & & \\
\hline \multicolumn{8}{|c|}{ Platelet-to-lymphocyte ratio } \\
\hline$<22,563.8$ & 10 & 1.00 & & - & 1.00 & & - \\
\hline $22,563.8-26,816.1$ & 6 & 1.50 & $0.51-4.41$ & 0.463 & 4.69 & $1.16-18.97$ & 0.030 \\
\hline \multirow[t]{2}{*}{$30,770-131,016$} & 7 & 2.94 & $0.95-9.09$ & 0.061 & 5.67 & $1.43-22.38$ & 0.013 \\
\hline & & $\mathrm{P}_{\text {trend }}=0.076$ & & & $P_{\text {trend }}=0.011$ & & \\
\hline \multicolumn{8}{|c|}{ Lymphocyte-to-monocyte ratio } \\
\hline$<2.7$ & 9 & 1.00 & & - & 1.00 & & - \\
\hline $2.7-4.2$ & 6 & 1.44 & $0.49-4.18$ & 0.507 & 0.24 & $0.07-0.84$ & 0.025 \\
\hline $4.3-6.3$ & 8 & 0.11 & $0.02-0.57$ & 0.008 & 0.14 & $0.04-0.56$ & 0.006 \\
\hline & & $\mathrm{P}_{\text {trend }}=0.015$ & & & $\mathrm{P}_{\text {trend }}=0.005$ & & \\
\hline LDH & & & & & & & \\
\hline $118-211$ & 17 & 1.00 & - & - & 1.00 & - & - \\
\hline $236-586$ & 7 & 3.09 & $1.06-8.98$ & 0.039 & 3.42 & $1.11-10.50$ & 0.032 \\
\hline CRP & & & & & & & \\
\hline$<0.18$ & 8 & 1.00 & - & - & 1.00 & - & - \\
\hline $0.18-1.14$ & 8 & 3.92 & $1.08-14.23$ & 0.038 & 1.18 & $0.31-4.48$ & 0.810 \\
\hline $1.25-5.10$ & 7 & 5.08 & $1.29-20.05$ & 0.020 & 10.58 & $2.25-49.89$ & 0.003 \\
\hline & & $\mathrm{P}_{\text {trend }}=0.028$ & & & $P_{\text {trend }}=0.007$ & & \\
\hline
\end{tabular}

Table 4. Exploratory analysis of the biomarkers. $H R$ hazard ratio, $C I$ confidence interval, irAE immunerelated adverse event, $P D-L 1$ programmed death-ligand 1, $m G P S$ modified Glasgow prognostic score, $L D H$ lactate dehydrogenase, CRP C-reactive protein. 
A

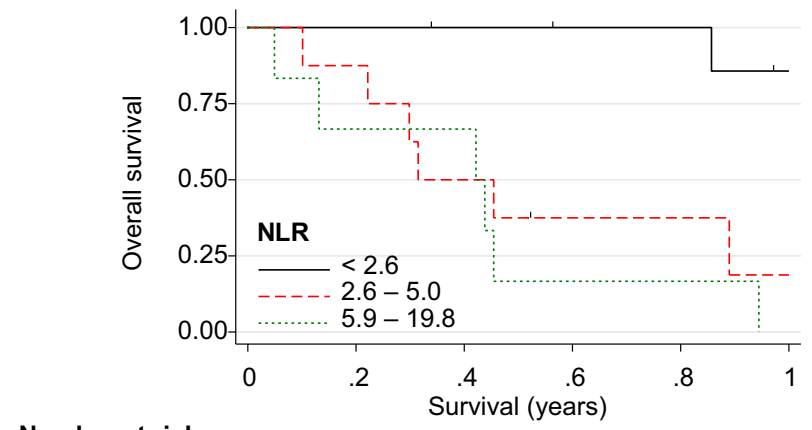

Number at risk

$\begin{array}{rrrrrrr}<2.6 & 9 & 9 & 8 & 7 & 7 & 5 \\ 2.6-5.0 & 8 & 7 & 4 & 2 & 2 & 1 \\ 5.9-19.8 & 6 & 4 & 4 & 1 & 1 & 0\end{array}$

C

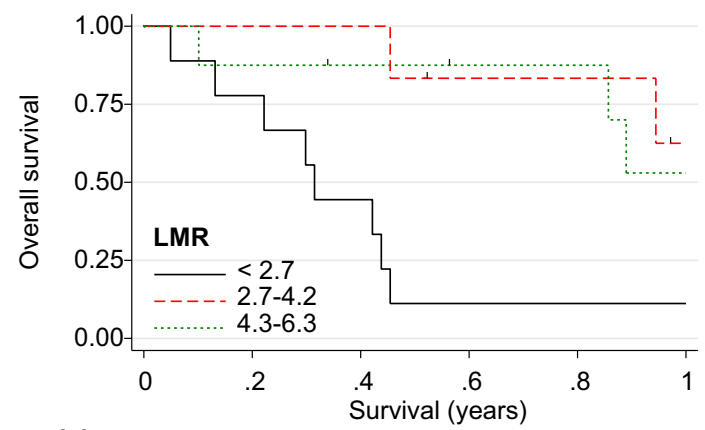

Number at risk

$\begin{array}{rllllll}<2.7 & 9 & 7 & 4 & 1 & 1 & 1 \\ 2.7-4.2 & 6 & 6 & 6 & 4 & 4 & 2 \\ 4.3-6.3 & 8 & 7 & 6 & 5 & 5 & 3\end{array}$

E

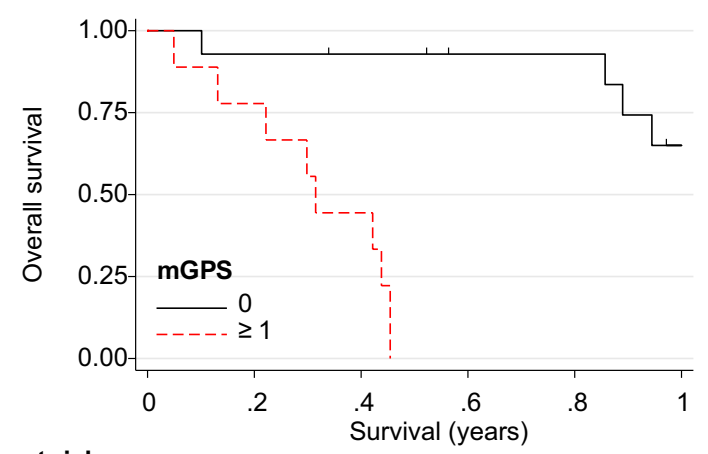

Number at risk

$\begin{array}{rcccccc}\text { risk } & & & & & 10 & 6 \\ 0 & 14 & 13 & 12 & 10 & 10 & 0\end{array}$

B

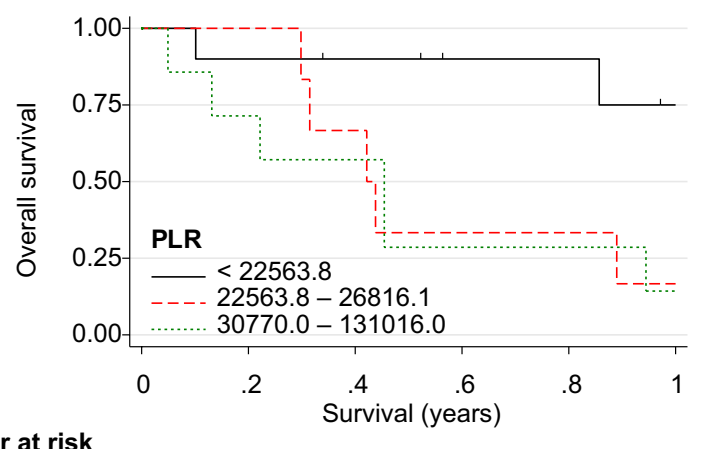

Number at risk

$\begin{array}{rllllll}<22563.8 & 10 & 9 & 8 & 6 & 6 & 4 \\ 22563.8-26816.1 & 6 & 6 & 4 & 2 & 2 & 1 \\ 30770.0-131016.0 & 7 & 5 & 4 & 2 & 2 & 1\end{array}$

D

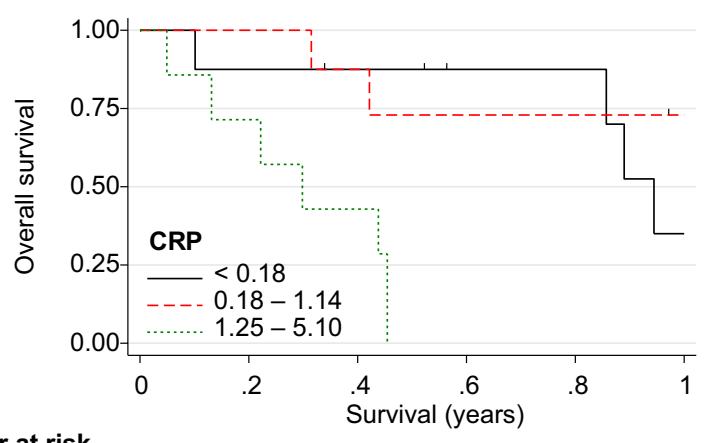

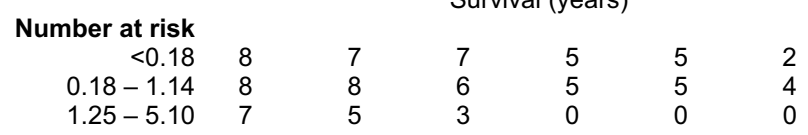

$\mathbf{F}$

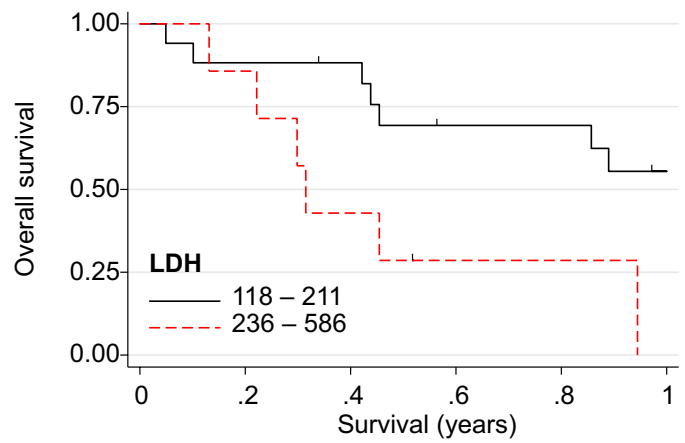



Figure 4. Kaplan-Meier curves of overall survival in patients with salivary gland carcinoma stratified by (A) the neutrophil-to-lymphocyte ratio (NLR), (B) platelet-to-lymphocyte ratio (PLR), (C) lymphocyte-tomonocyte ratio (LMR), (D) C-reactive protein (CRP), (E) modified Glasgow prognostic score (mGPS), and (F) lactate dehydrogenase (LDH) activity.

response to nivolumab. Moreover, the optimal cut-off value for NLR was unknown. Thus, future clinical trials with a larger sample size should be performed to address these issues.

In the present study, the efficacy of nivolumab monotherapy for SGC was limited. However, some patients achieved long-term disease control with nivolumab. Further studies are warranted to elucidate a predictive factor of ICI in patients with advanced SGC. 


\section{Materials and methods}

Patients and treatment. This was a multicentre retrospective cohort study conducted in Japan. Following approval from the ethics committee of the participating institutions (Approval number of each institution: International University of Health and Welfare, Mita Hospital, 5-18-50; Nihonkai General Hospital, 30-(4)-3; Niigata University, 2019-0056; Tokyo Medical University, T2018-0059; Nagoya City University, 60-20-0049), data of patients with unresectable R/M SGC treated with nivolumab between May 2017 and September 2019 were extracted from the database of the nation-wide cancer registry of each participating institution. This study was conducted in accordance with the Declaration of Helsinki. Written informed consent was obtained from each patient and/or their legal guardians. Additionally, we obtained informed consent of the patients for publication of identifying images and photographs.

Patients with $\geq 20 \%$ tumour growth within 6 months prior to treatment detected by computed tomography (CT) scan, magnetic resonance imaging, and/or positron emission tomography-CT were treated with nivolumab (240 mg) every 2 weeks. The treatment dose and duration were determined in accordance with the Japanese guidelines for head and neck cancer, including salivary gland cancer. A pathological review of all patients was performed by a pathologist with expertise in SGCs (T.N.). Carcinoma ex pleomorphic adenomas were classified into different histopathological types according to each carcinoma component instead of a separate category. Imaging tests were performed every 6-8 weeks.

Immunohistochemical and gene alteration analyses. The expression status of PD-L1, MSI, HER2, and androgen receptor (AR) in patients administered nivolumab was also obtained from the database of the participating institutes. The expression level of PD-L1 in the resected or biopsy specimens of tumours was analysed using the rabbit antihuman PD-L1 clone 28-8 using the automated immunohistochemical assay (PDL1 IHC 28-8 pharmDx; Dako-Agilent Technologies, Santa Clara, CA, USA). PD-L1 expression was defined as the percentage (instead of intensity) of tumour cells exhibiting plasma membrane staining 22 .

The MSI test kit (product code: 4987931010017; FALCO Biosystems, Kyoto, Japan) was used to evaluate MSI as described previously ${ }^{54}$. Briefly, a polymerase chain reaction (PCR) of microsatellite markers at five loci (BAT25, BAT26, NR21, NR24, and MONO27) was conducted using DNA extracted from tumour specimens. In normal patients, the PCR products were in the quasi-monomorphic variation range (QVR). Specimens with the PCR products outside the QVR were classified as MSI-positive. Specimens with more than one positive locus were classified as MSI-high (MSI-H).

HER2 and AR statuses were assessed as described previously ${ }^{5,10,55,56}$. Briefly, specimens with $3+$ HER 2 immunoreactivity or HER2 gene amplification were classified as HER2-positive according to the guidelines for breast cancer by the American Society of Clinical Oncology and the College of American Pathologists ${ }^{56}$. AR was classified as positive if $\geq 20 \%$ of nuclei in tumour cells were immunoreactive.

Analysis of biomarkers of ICI response. An exploratory analysis of potential biomarkers of ICI response was performed ${ }^{43,46}$. The associations between prognosis and age, sex, the Eastern Cooperative Oncology Group (ECOG) performance status (PS), prior systemic therapy (present or absent), immune-related adverse events (irAE; present or absent), systemic therapy after nivolumab (present or absent), histopathological type (SDC or non-SDC), PD-L1 status, HER2 status, AR status, MSI status, mGPS, NLR, PLR, LMR, serum CRP, LDH, and absolute eosinophil count were examined.

Statistical analysis. The therapeutic effect of nivolumab was evaluated according to the overall response rate (ORR), which was defined as the percentage of patients who achieved complete response (CR) or partial response (PR), clinical benefit rate [CBR, defined as the percentage of patients who achieved CR, $P R$, or stable disease (SD) for at least 24 weeks], disease control rate (DCR, defined as the percentage of patients who achieved $\mathrm{CR}, \mathrm{PR}$, or SD regardless of duration), median progression-free survival (PFS), and median overall survival $(\mathrm{OS})^{5,10,43,46}$. Treatment efficacy was evaluated according to Response Evaluation Criteria in Solid Tumors version 1.1 (RECIST 1.1) ${ }^{57}$. PFS was defined as the time from the start of nivolumab treatment to the diagnosis of progressive disease (PD). OS was defined as the time from the start of nivolumab treatment to death from any cause. Safety was evaluated according to Common Terminology Criteria for Adverse Events (CTCAE) ver. $5.0^{58}$. The Kaplan-Meier method was used to estimate PFS and OS. The Cox proportional hazards model was used to calculate the hazard ratio (HR) with $95 \%$ confidence interval (CI). The results with a $P$ value of $<0.05$ were considered statistically significance. All analyses were performed using STATA ver. 16 (StataCorp, College Station, TX, USA).

\section{Data availability}

The datasets generated in the current study are available from the corresponding author on request.

Received: 8 June 2020; Accepted: 22 September 2020

Published online: 12 October 2020

\section{References}

1. Cancer Registry and Statistics. Cancer Information Service, National Cancer Center, Japan (Ministry of Health, Labour and Welfare, National Cancer Registry.) https://ganjoho.jp/data/reg_stat/statistics/dl/cancer_incidenceNCR(2016-2017).xls. Accessed 28 September 2020.

2. El-Naggar, A. K. et al. WHO Classification of Head and Neck Tumours 4th edn. (IARC, Lyon, 2017). 
3. Lewis, A. G., Tong, T. \& Maghami, E. Diagnosis and management of malignant salivary gland tumors of the parotid gland. Otolaryngol. Clin. N. Am. 49, 343-380 (2016).

4. Uijen, M. J. M. et al. Systemic therapy in the management of recurrent or metastatic salivary duct carcinoma: A systematic review. Cancer Treat. Rev. https://doi.org/10.1016/j.ctrv.2020.102069 (2020).

5. Takahashi, H. et al. Phase II trial of trastuzumab and docetaxel in patients with human epidermal growth factor receptor 2-positive salivary duct carcinoma. J. Clin. Oncol. 37, 125-134 (2019).

6. Jhaveri, K. L. et al. Ado-trastuzumab emtansine (T-DM1) in patients with HER2-amplified tumors excluding breast and gastric/ gastroesophageal junction (GEJ) adenocarcinomas: Results from the NCI-MATCH trial (EAY131) subprotocol Q. Ann. Oncol. 30, 1821-1830 (2019).

7. Kurzrock, R. et al. Targeted therapy for advanced salivary gland carcinoma based on molecular profiling: Results from MyPathway, a phase IIa multiple basket study. Ann. Oncol. 31, 412-421 (2020).

8. Tsurutani, J. et al. Targeting HER2 with trastuzumab deruxtecan: A dose-expansion, phase i study in multiple advanced solid tumors. Cancer Discov. 10, 688-701 (2020).

9. Locati, L. D. et al. Clinical activity of androgen deprivation therapy in patients with metastatic/relapsed androgen receptor-positive salivary gland cancers. Head Neck. 38, 724-731 (2016).

10. Fushimi, C. et al. A prospective phase II study of combined androgen blockade in patients with androgen receptor-positive metastatic or locally advanced unresectable salivary gland carcinoma. Ann. Oncol. 29, 979-984 (2018).

11. Boon, E. et al. Androgen deprivation therapy for androgen receptor-positive advanced salivary duct carcinoma: A nationwide case series of 35 patients in The Netherlands. Head Neck. 40, 605-613 (2018).

12. Viscuse, P. V. et al. First line androgen deprivation therapy vs. chemotherapy for patients with androgen receptor positive recurrent or metastatic salivary gland carcinoma-A retrospective study. Front. Oncol. 9, 701 (2019).

13. Hong, D. S. et al. Larotrectinib in patients with TRK fusion-positive solid tumours: A pooled analysis of three phase $1 / 2$ clinical trials. Lancet Oncol. 21, 531-540 (2020).

14. Doebele, R. C. et al. Entrectinib in patients with advanced or metastatic NTRK fusion-positive solid tumours: Integrated analysis of three phase 1-2 trials. Lancet Oncol. 21, 271-282 (2020).

15. Tchekmedyian, V. et al. Phase II study of lenvatinib in patients with progressive, recurrent or metastatic adenoid cystic carcinoma. J. Clin. Oncol. 37, 1529-1537 (2019).

16. Locati, L. D. et al. Patients with adenoid cystic carcinomas of the salivary glands treated with lenvatinib: Activity and quality of life. Cancer 126, 1888-1894 (2020).

17. Ferrarotto, R. et al. Vergara-Silva. ACCURACY a phase (P) II trial of AL101, a pan-Notch inhibitor, in recurrent/metastatic (R/M) adenoid cystic carcinoma (ACC) patients (pts) with Notch activating mutations (Notch act mut): Preliminary safety and efficacy data. Ann. Oncol. 30, v465-v466 (2019).

18. Mimica, X. et al. Distant metastasis of salivary gland cancer: Incidence, management, and outcomes. Cancer 126, 2153-2162 (2020).

19. Hanna, G. J. et al. Long-term outcomes and clinicogenomic correlates in recurrent, metastatic adenoid cystic carcinoma. Oral Oncol. 106, $104690(2020)$.

20. Nagao, T., Licitra, L., Loening, T., Vielh, P. \& Williams, M. D. Salivary duct carcinoma. In WHO Classification of Head and Neck Tumours 4th edn (eds Naggar, A. K. et al.) 173-174 (Lyon, IARC, 2017).

21. Nakaguro, M. et al. Salivary duct carcinoma: Updates in histology, cytology, molecular biology, and treatment. Cancer Cytol. https ://doi.org/10.1002/cncy.22288 (2020).

22. Ferris, R. L. et al. Nivolumab for recurrent squamous-cell carcinoma of the head and neck. N. Engl. J. Med. 375, 1856-1867 (2016).

23. Burtness, B. et al. Pembrolizumab alone or with chemotherapy versus cetuximab with chemotherapy for recurrent or metastatic squamous cell carcinoma of the head and neck (KEYNOTE-048): A randomised, open-label, phase 3 study. Lancet 394, 1915-1928 (2019).

24. Cohen, R. B. et al. Pembrolizumab for the treatment of advanced salivary gland carcinoma: Findings of the phase $1 \mathrm{~b}$ KEYNOTE-028 study. Am. J. Clin. Oncol. 41, 1083-1088 (2018).

25. Rodriguez, C. P. et al. A Phase II Trial of pembrolizumab and vorinostat in recurrent metastatic head and neck squamous cell carcinomas and salivary gland cancer. Clin. Cancer Res. 26, 837-845 (2020).

26. Hori, R. et al. Real-world outcomes and prognostic factors in patients receiving nivolumab therapy for recurrent or metastatic head and neck carcinoma. Cancers (Basel). https://doi.org/10.3390/cancers11091317 (2019).

27. Kokkali, S. et al. Nivolumab in patients with rare head and neck carcinomas: A single center's experience. Oral Oncol. 101, 104359 (2020).

28. Topalian, S. L., Taube, J. M., Anders, R. A. \& Pardoll, D. M. Mechanism-driven biomarkers to guide immune checkpoint blockade in cancer therapy. Nat. Rev. Cancer. 16, 275-287 (2016).

29. Ott, P. A. et al. T-Cell-inflamed gene-expression profile, programmed death ligand 1 expression, and tumor mutational burden predict efficacy in patients treated with pembrolizumab across 20 cancers: KEYNOTE-028. J. Clin. Oncol. 37, 318-327 (2019).

30. Le, D. T. et al. PD-1 blockade in tumors with mismatch-repair deficiency. N. Engl. J. Med. 372, 2509-2520 (2015)

31. Rizvi, N. A. et al. Cancer immunology. Mutational landscape determines sensitivity to PD-1 blockade in non-small cell lung cancer. Science 348, 124-128 (2015).

32. Ho, A. S. et al. Genetic hallmarks of recurrent/metastatic adenoid cystic carcinoma. J. Clin. Investig. 129, 4276-4289 (2019).

33. Nakano, T. et al. Prognostic value of programed death ligand-1 and ligand-2 co-expression in salivary gland carcinomas. Oral Oncol. 90, 30-37 (2019).

34. Gargano, S. M. et al. Novel therapeutic targets in salivary duct carcinoma uncovered by comprehensive molecular profiling. Cancer Med. 8, 7322-7329 (2019).

35. Mukaigawa, T. et al. Programmed death ligand-1 expression is associated with poor disease free survival in salivary gland carcinomas. J. Surg. Oncol. 114, 36-43 (2016).

36. Chang, H. et al. Overexpression of PD-L2 is associated with shorter relapse-free survival in patients with malignant salivary gland tumors. Oncol. Targets Ther. 10, 2983-2992 (2017).

37. Szewczyk, M. et al. Prognostic markers in salivary gland cancer and their impact on survival. Head Neck. 41, 3338-3347 (2019).

38. Vital, D. et al. The expression of PD-L1 in salivary gland carcinomas. Sci. Rep. 9, 12724 (2019).

39. Linxweiler, M. et al. The immune microenvironment and neoantigen landscape of aggressive salivary gland carcinomas differ by subtype. Clin. Cancer Res. https://doi.org/10.1158/1078-0432.CCR-19-3758 (2020).

40. Dalin, M. G. et al. Comprehensive molecular characterization of salivary duct carcinoma reveals actionable targets and similarity to apocrine breast cancer. Clin. Cancer Res. 22, 4623-4633 (2016).

41. Ross, J. S. et al. Comprehensive genomic profiles of metastatic and relapsed salivary gland carcinomas are associated with tumor type and reveal new routes to targeted therapies. Ann. Oncol. 28, 2539-2546 (2017).

42. Terzić, J., Grivennikov, S., Karin, E. \& Karin, M. Inflammation and colon cancer. Gastroenterology 138, 2101-2114.e5 (2010).

43. Kawakita, D. et al. Impact of hematological inflammatory markers on clinical outcome in patients with salivary duct carcinoma: A multi-institutional study in Japan. Oncotarget. 8, 1083-1091 (2017).

44. Templeton, A. J. et al. Prognostic role of neutrophil-to-lymphocyte ratio in solid tumors: A systematic review and meta-analysis. J. Natl Cancer Inst. 106, dju124 (2014). 
45. Ferrucci, P. F. et al. Baseline neutrophils and derived neutrophil-to-lymphocyte ratio: Prognostic relevance in metastatic melanoma patients receiving ipilimumab. Ann. Oncol. 27, 732-738 (2016).

46. Matsuki, T. et al. Hematological predictive markers for recurrent or metastatic squamous cell carcinomas of the head and neck treated with nivolumab: A multicenter study of 88 patients. Cancer Med. https://doi.org/10.1002/cam4.3124 (2020).

47. Martens, A. et al. Baseline peripheral blood biomarkers associated with clinical outcome of advanced melanoma patients treated with ipilimumab. Clin. Cancer Res. 22, 2908-2918 (2016).

48. Yamamoto, Y. et al. Prognostic value of risk stratification using blood parameters for nivolumab in Japanese patients with metastatic renal-cell carcinoma. Jpn J. Clin. Oncol. 50, 214-220 (2020).

49. Diem, S. et al. Neutrophil-to-lymphocyte ratio (NLR) and platelet-to-lymphocyte ratio (PLR) as prognostic markers in patients with non-small cell lung cancer (NSCLC) treated with nivolumab. Lung Cancer. 111, 176-181 (2017).

50. Delyon, J. et al. Experience in daily practice with ipilimumab for the treatment of patients with metastatic melanoma: An early increase in lymphocyte and eosinophil counts is associated with improved survival. Ann. Oncol. 24, 1697-1703 (2013).

51. Tanizaki, J. et al. Peripheral blood biomarkers associated with clinical outcome in non-small cell lung cancer patients treated with nivolumab. J. Thorac. Oncol. 13, 97-105 (2018).

52. Seiwert, T. Y. et al. Safety and clinical activity of pembrolizumab for treatment of recurrent or metastatic squamous cell carcinoma of the head and neck (KEYNOTE-012): An open-label, multicentre, phase 1b trial. Lancet Oncol. 17, 956-965 (2016).

53. Ferris, R. L. et al. Abstract CT021: Tumor-associated immune cell PD-L1 expression and peripheral immune profiling: Analyses from CheckMate 141. Cancer Res. 77, CT021 (2017).

54. Buhard, O. et al. Multipopulation analysis of polymorphisms in five mononucleotide repeats used to determine the microsatellite instability status of human tumors. J. Clin. Oncol. 24, 241-251 (2006).

55. Takase, S. et al. Biomarker immunoprofile in salivary duct carcinomas: Clinicopathological and prognostic implications with evaluation of the revised classification. Oncotarget. 8, 59023-59035 (2017).

56. Wolff, A. C. et al. Recommendations for human epidermal growth factor receptor 2 testing in breast cancer: American Society of Clinical Oncology/College of American Pathologists clinical practice guideline update. J. Clin. Oncol. 31, 3997-4013 (2013).

57. Eisenhauer, E. A. et al. New response evaluation criteria in solid tumours: Revised RECIST Guideline (Version 1.1). Eur. J. Cancer. 45, 228-247 (2009).

58. Common Terminology Criteria for Adverse Events (CTCAE) v5.0. https://ctep.cancer.gov/protocolDevelopment/electronic_appli cations/ctc.htm\#ctc_50.

\section{Acknowledgements}

We thank all patients and their families and caregivers. This work was supported by the JSPS Grant-in-Aid for Scientific Research (C) to Dr. Yuichiro Tada [Grant number 18K09386], Dr. Daisuke Kawakita [Grant number 20K10508], Dr. Toshitaka Nagao [Grant number 17K08705], Dr. Hideaki Hirai [Grant number 19K16568], Dr. Hideaki Takahashi [Grant number 19K09873], and Dr. Takashi Matsuki [Grant number 20K07597].

\section{Author contributions}

Study concept and design: Y.T., I.O., C.F., T.M., K.T. and N.O.; acquisition of data: Y.T., K.N., T.S., M.O., K.Y., I.O., T.O., H.H., N.S., T.M., K.M., K.H., S.O, H.M., and S.I.; analysis and interpretation of data: D.K., K.N., and Y.T.; statistical analysis: D.K., K.N., and Y.T.; drafting of the manuscript: K.N., Y.T., D.K., T.N., and H.T.; manuscript review: all authors.

\section{Competing interests}

The authors declare no competing interests.

\section{Additional information}

Supplementary information is available for this paper at https://doi.org/10.1038/s41598-020-73965-6.

Correspondence and requests for materials should be addressed to Y.T.

Reprints and permissions information is available at www.nature.com/reprints.

Publisher's note Springer Nature remains neutral with regard to jurisdictional claims in published maps and institutional affiliations.

Open Access This article is licensed under a Creative Commons Attribution 4.0 International License, which permits use, sharing, adaptation, distribution and reproduction in any medium or format, as long as you give appropriate credit to the original author(s) and the source, provide a link to the Creative Commons licence, and indicate if changes were made. The images or other third party material in this article are included in the article's Creative Commons licence, unless indicated otherwise in a credit line to the material. If material is not included in the article's Creative Commons licence and your intended use is not permitted by statutory regulation or exceeds the permitted use, you will need to obtain permission directly from the copyright holder. To view a copy of this licence, visit http://creativecommons.org/licenses/by/4.0/.

(C) The Author(s) 2020 